\title{
Intelligent Bioproduction System towards Environment Protection
}

\author{
Noboru NoGUCHI \\ Bioproduction Engineering, Graduate School of Agriculture, Hokkaido University, \\ Kita-9, Nishi-9, Kita-ku, Sapporo, Hokkaido 060-8589, Japan
}

(Received March 18, 2009)

\begin{abstract}
A complex interaction involving temporal as well as spatial factors influences plant growth and development. To optimally control the environment for plant growth, an important first step is to develop crop growth models that can predict daily plant growth based on weather data, management practices, and plant genetic information. When this is accomplished, sensing data can contribute to enhanced accuracy of the plant growth model. Recent efforts to integrate plant growth models with sensing methods have provided an opportunity to optimize future plant production systems. In other words, good results require feedback from plants. The general concept is coined the 'speaking plant' concept. Consequently, sensors are an essential part of control systems. Machine vision can provide information about current crop status, including growth, nutrient stress and pest infestation. In this paper, current technologies are introduced and sensing system using artificial intelligence are described.
\end{abstract}

Keywords : artificial intelligence, fuzzy logic, genetic algorithms, neural networks, machine vision

\section{INTRODUCTION}

Agriculture methods in developed countries since the Industrial Revolution have tended to favor greater energy inputs using large tractors and increased applications of synthetic chemicals and fertilizer. While these methods of agricultural production have some negative societal and environmental implications, they have generally supported the food needs of the rapidly growing human population. However, a new mode of thought, with new technology is needed for the future. An agricultural production system that utilizes recent advances in sensor technology can fill this role, becoming an essential component of an intelligent agricultural production system. The trend towards intelligent agricultural production systems will firstly lead to information agriculture as a specific application of the field of infotronics in the agriculture sector. Infotronics is the integration of electronics, computing and information technologies to achieve functional operations under the dynamic changes and needs of a real environment (Fig. 1). Infotronics can expand the capabilities of precision agriculture to maximize agriculture profitability and minimize consumption of resources through optimizing agricultural production based on information from in-field and, more importantly, off-field sources. Remote sensing has recently been regarded as a promising source of information about current crop status, including maturity and weed infestations. An intelligent machine vision system for an agricultural robot, as shown in Fig. 2, is also promising technology for production systems in the future. A vision system is able to simultaneously detect crop rows and gather field information. Nitrogen stress of the crop is one of the most important factors

Corresponding author: Noboru Noguchi, fax : +81-11-706-4800, e-mail : noguchi@bpe.agr.hokudai.ac.jp 


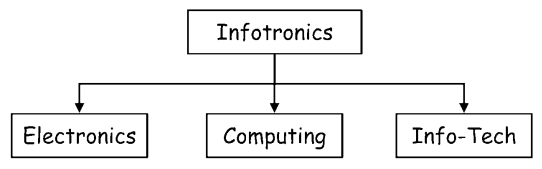

Fig. 1 Infotronics integrates sensors and information through computer technology.

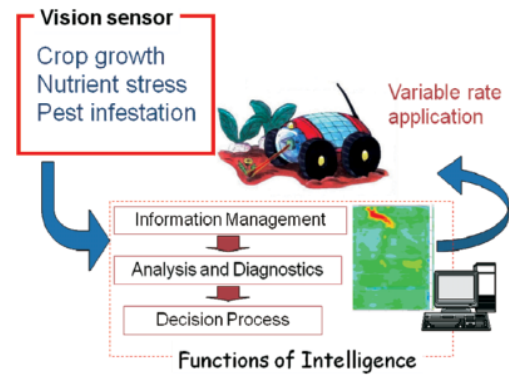

Fig. 2 Concept of vision intelligence for agricultural robot.

for crop growth and yield. A sensor for detecting the crop status must adopt a non-contact base method. An intelligent vision system based on a multi-spectral imaging system was developed for detecting nitrogen stress and crop height by collaboration of Hokkaido University and University of Illinois (Noguchi et al., 2001). The vision system enables a robot to recognize the crop status and efficiently conduct field operations through timely information. Outputs include crop stress maps with nitrogen deficiency indexes on the field. As the next step, decision-making by the robot itself regarding fertilizer treatment is required for real-time nitrogen application. Sensing technology can contribute to precision farming and identification of a crop growth model as a complex system. Obviously, this kind of sensing system can be also applied to greenhouse cultivation (Carson et al., 2008). Figure 3 shows an example of intelligent control systems for greenhouse cultivation based on crop sensing and optimal control (van Straten et al., 2008). Whatever the control method is, good results require feedback from plants. The general concept is sometimes coined the 'speaking plant' or 'speaking fruit' concept (Hashimoto et al., 2006). In the past, modeling and control based on artificial intelligence were applied to a fruit-storage process, and obtained suboptimal solutions. The results were satisfactory (Morimoto et al., 1997). Consequently, sensors are an essential part of intelligent systems. This paper introduces current technologies and control systems using tools categorized as artificial intelligence (AI) such as fuzzy logic (FL), artificial neural networks (ANN) and genetic algorithms (GA), and their impact on agricultural production systems is discussed.

\section{ARTIFICIAL INTELLIGENCE}

In the past decades, there have been attempts to use various types of AI tools such as expert systems, artificial neural networks, genetic algorithms, simulated annealing and data mining in bioproduction systems. Usually, AI has two main goals. One is to understand human intelligence better. In the past, researchers tested theories of human intelligence by writing programs that emulate it. Another is to create useful "smart" programs that are able to do tasks that would normally require a human expert. Popular AI tools such as FL, ANN and GA are briefly explained here. The FL theory was first proposed by Zadeh in 1965. Its greatest advantage is that it is very useful for analysis when there is no reliable system model. Because accurate dynamic equations of systems 


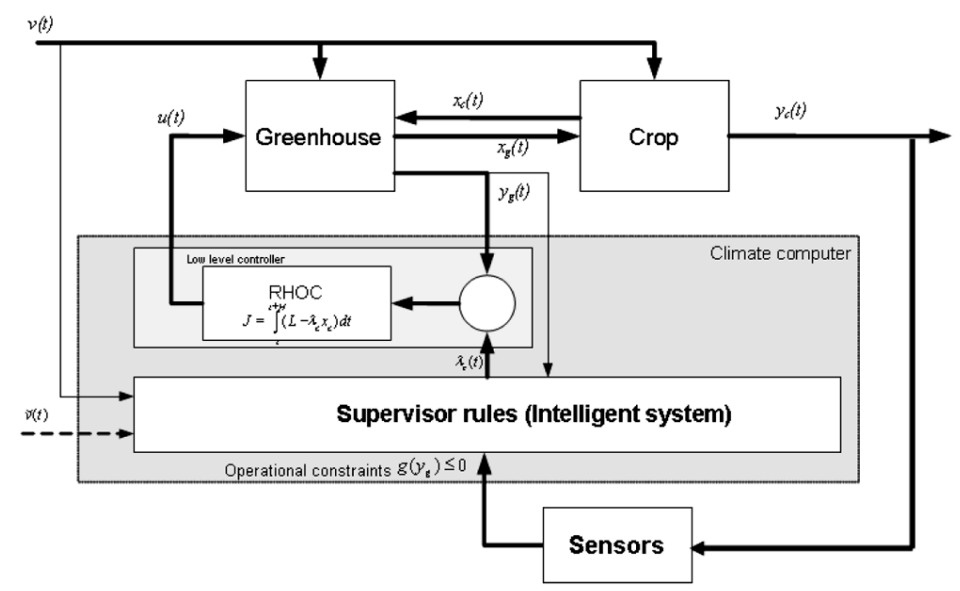

Fig. 3 Intelligent control system on greenhouse cultivation. (van Straten and van Willigenburg, 2008)

cannot be easily developed, FL has become popular in studies on complex systems. Conventionally, FL is based on expert knowledge to define input-output variables, linguistic variables, fuzzy rules, and universal ranges.

On the other hand, ANN-based black-box models have high mapping capabilities and guarantee a good generalization even with a reduced set of identification data. Moreover, the possibility of implementing adaptive training procedures allows the influence of exogenous effects on control performance to be taken into account.

In computer science, evolutionary computation is a subfield of artificial intelligence involving combinatorial optimization problems (Holland, 1962). Evolutionary computation includes genetic algorithms (GA), evolutionary programming (EP), evolution strategies (ES), genetic programming (GP), and classifier systems (CS). The GA, which is an optimization and search technique based on the principles of genetics and natural selection, was proposed by John Holland in 1962. By 1975, Holland reported the most basic principle of the GA in "Adaptation in Natural and Artificial System" (Holland, 1975). The GA generally only involves techniques of implementing mechanisms, such as reproduction, crossover, mutation, and fittest function. The GA can generate the next generation to reach the purpose of evolution via reproduction, crossover, and mutation steps. According to the level of fitness, the GA retains the fine individuals (chromosome) and eliminates inferior individuals. Therefore, it has been used widely to solve optimal problems recently. It can search many points at the same time and is not apt to fall into a local optimal solution because of an operator of the mutation. The GA has been used for a wide range of applications as well as for specific applications focused on a specific requirement (Noguchi and Terao, 1997).

\section{DEVELOPMENT OF A "SMART” VISION SENSOR FOR A FIELD ROBOT}

Knowledge of the growers, which is rule-based approach, sometimes becomes a powerful tool for perception and control in agriculture. However, it is difficult to implement and code human intelligence. The performance of AI mostly depends on the skill of a system designer (developer). Sometimes there are no sensors to detect the expert's sensory feeling. Therefore, automatic creation of a control strategy as well as wisdom and knowledge generated by the actual plant data which does not depend on a human expert are strongly desired for maintaining and generalizing system performance. The developed vision system uses a GA-optimized FL decision-making system to classify crop and weed materials. After segmenting out the weed, the "Smart" vision system 
can estimate the crop height and width using the ANN (Noguchi et al., 1999).

Classification of crop and weeds by a combination of FL and GA

The machine vision system can quickly gather a large amount of information in a short period of time. To reduce the processing load and to decrease the computer memory requirements, images are compressed by expressing the canopy area as a density within a $20-\mathrm{cm}$ square. The upper image in Fig. 4 was obtained through binalization of a raw image in a $4 \mathrm{~m} \times 2 \mathrm{~m}$ field of view. The lower image in Fig. 4 shows the canopy area represented as a gray level calculated through the transformation mentioned above. Because the actual area of each pixel is spatially different, the conversion coefficient from pixel size to actual area was calculated for each of the 20 -cm squares.

FL, which can deal with ambiguous information, was used as the crop and weed classifier in this study. FL can represent the solution in terms of probability. Therefore, three fuzzy input parameters, $x_{I}$ to $x_{3}$, were chosen to classify crop and weed areas in the field of view. Since crop rows are almost parallel to the $y$-axis of the field of view during run, we chose the average of the gray level for each row in the image as $x_{1}$, the standard deviation of that as $x_{2}$, and the spatial weighting factor as $x_{3}$. As shown in Fig. 5, the spatial weighting factor, $x_{3}$, utilized the fixed crop row width as priori information. The FL input parameters are normalized and can be expressed as follows:

$$
\begin{gathered}
x_{1}=\sum_{j} g_{i, j} / n_{j} \\
x_{2}=100 \sqrt{\frac{\sum_{j}\left(g_{j}-x_{1}\right)^{2}}{n} / x_{1}}
\end{gathered}
$$

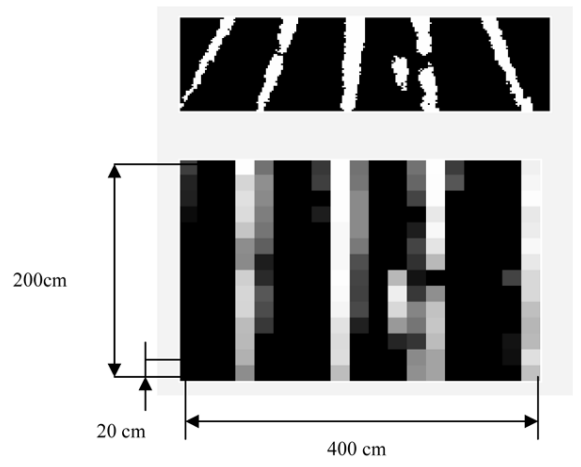

Fig. 4 Conversion and compression of the image information.

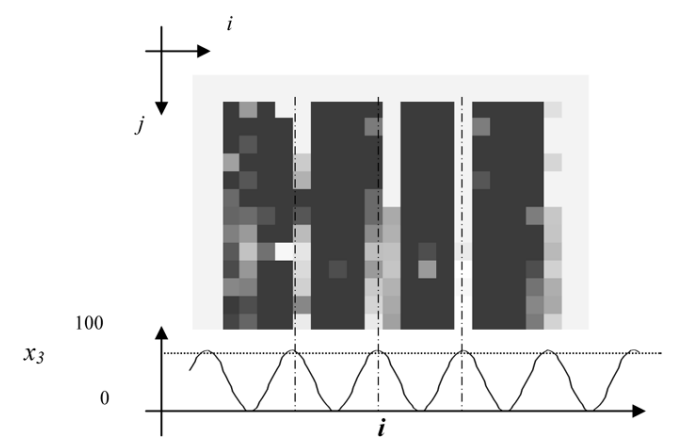

Fig. 5 Definition of spatial weighting factor, $x_{3}$. 


$$
x_{3}=50 \sin (i-\pi)(2 \pi / 4.5)+50
$$

The input and output membership functions and the fuzzy rules are shown in Fig. 6, Fig. 7 and Table 1. The FL was designed so as to output the possibility of crop from the FL classifier. One of the disadvantages of FL compared to a conventional image classifier (such as the K-means algorithm or a simple threshold-based segmentation) is that classification accuracy depends on the shape of the membership functions and the fuzzy rules built by a designer. Trial-and-error methods are frequently used to determine the FL parameters and membership functions. A GA was used to optimize the FL input membership functions, the fuzzy rules and the output membership functions. In this study, coefficients $a_{i}$ and $b_{i}\left(i=0\right.$ to 5 ) in the input membership function and $p_{0}$ to $p_{3}$ in the output membership function, which decide the shape of those, were coded in individuals as shown in Fig. 8. The flowchart of the GA for optimizing the FL classifier is shown in Fig. 9. Crossover was a random exchange of multiple alleles between the selected mating pairs, which created two offspring. Half of an individual's total number of alleles was exchanged during crossover. Mutation occurred with a probability of 1.0 in selected individuals that had not crossed over. All of the alleles were increased by a mutation width $\Delta \varepsilon$ that was randomly chosen in the range of -10 to 10 . After these transitional operators created new individuals, the survivors were selected

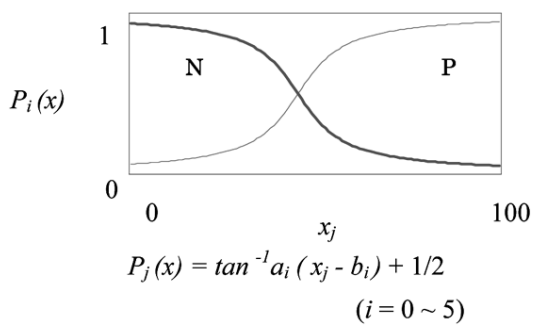

Fig. 6 Input membership function.

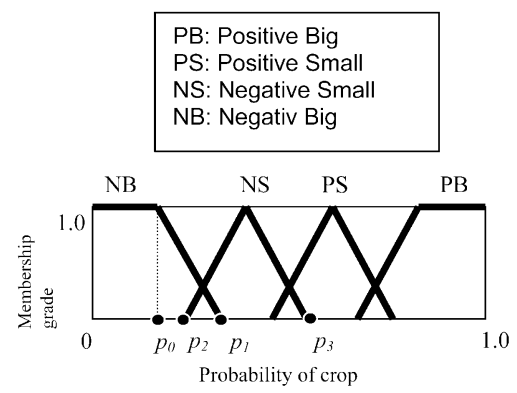

Fig. 7 Output membership function.

Table 1 Temporally fuzzy rules.

Rule 1: IF Input $x_{1}$ is P, Input $x_{2}$ is P, and Input $x_{3}$ is P THEN the class is PS.

Rule 2: IF Input $x_{1}$ is $\mathrm{P}$, Input $x_{2}$ is $\mathrm{P}$, and Input $x_{3}$ is N THEN the class is NS.

Rule 3: IF Input $x_{1}$ is $\mathrm{P}$, Input $x_{2}$ is $\mathrm{N}$, and Input $x_{3}$ is P THEN the class is PB.

Rule 4: IF Input $x_{1}$ is P, Input $x_{2}$ is $\mathrm{N}$, and Input $x_{3}$ is N THEN the class is PS.

Rule 5: IF Input $x_{1}$ is N, Input $x_{2}$ is $\mathrm{P}$, and Input $x_{3}$ is P THEN the class is NS.

Rule 6: IF Input $x_{1}$ is N, Input $x_{2}$ is $\mathrm{P}$, and Input $x_{3}$ is N THEN the class is NB.

Rule 7: IF Input $x_{1}$ is N, Input $x_{2}$ is $\mathrm{N}$, and Input $x_{3}$ is P THEN the class is PS.

Rule 8: IF Input $x_{1}$ is N, Input $x_{2}$ is N, and Input $x_{3}$ is N THEN the class is NS. 


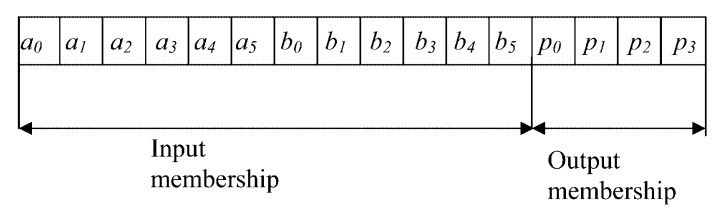

Fig. 8 Coding of the fuzzy parameters to a chromosome.

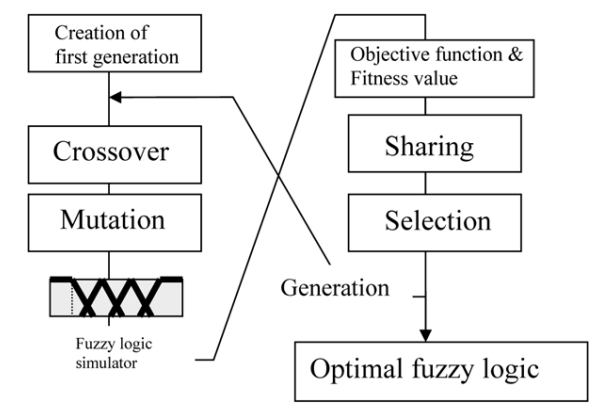

Fig. 9 Flowchart of GA optimization of fuzzy logic.

from the double-sized tentative population that included the current and newly created individuals. Selection, an analog to natural selection, was conducted by spinning a simulated roulette wheel whose slots had different sizes proportional to the fitness values of the individuals and by an elitist preservation strategy in which the individual with the lowest objective function value was exceptionally chosen. As shown in Eqn. (4), the adopted objective function was the error function summing up the squared error between the training data and the data calculated by each individual for all acquired image data.

$$
\mathrm{H} V\left(c_{i}\right)=\sum_{j=0}^{n}\left[\hat{P}_{j}-P\left(c_{i}, j\right)\right]^{2}
$$

Here,

$V$ : Objective function, P: FL possibility,

$C_{i}$ : i-th chromsome, $n$ : Number of training data,

$\widehat{P}$ : Real possibility.

The training data, including the real possibility of the crop, was determined manually. For the image data gathered over the entire growth period, $n$ was 1235 . The fitness value $f$ for each individual was converted from the objective function $V(u)$. In addition, to maintain the diversity of individuals in the evolution process, the fitness value was corrected using a sharing operation proposed by Ichikawa and Sano (1992). The size of the population for the next generation was also kept constant by choosing half of the tentative population. Results were obtained using the GA parameters of population size $m=20$, number of generations $N_{g}=500$, probability of crossover $P_{c}=$ 0.7 , and probability of mutation $P_{m}=0.3$. William 82 soybeans were chosen as the test crop in this study. The input and output membership functions created by the GA are shown in Fig. 10 and Fig. 11, respectively. The shapes of the membership functions were quite different from FL functions conventionally created by a human. In particular, the GA-created unsymmetrical membership seems unique in general FL. Figure 12 shows results of segmenting crop and weeds by the created FL. The top figure is a raw image and gray level in the second and third figures indicate the existence probabilities of crop and weeds. It was clear that the FL could correctly distinguish crops and weeds in the figure. Accuracy of the segmentation was investigated using images acquired in six different growth stages covering the entire growth period to verify robustness of the created FL 

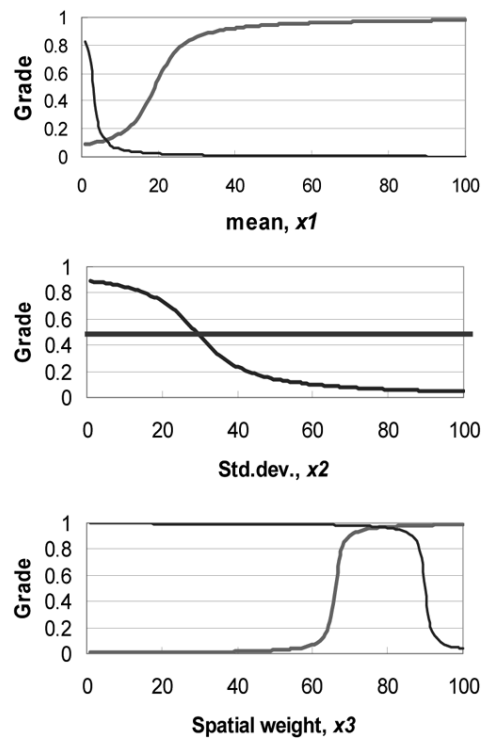

Fig. 10 Input membership function created by the GA.

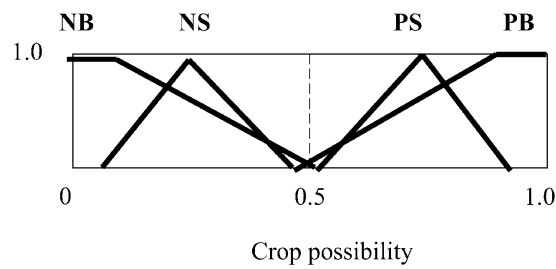

Fig. 11 Output membership function created by the GA.

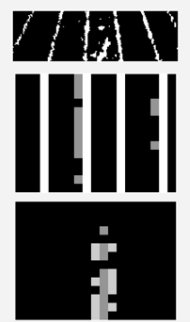

Raw

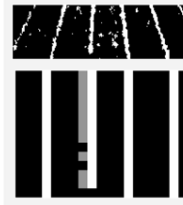

Crop

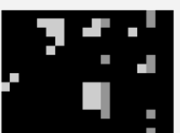

Fig. 12 Results using fuzzy logic created by GA.

(Fig. 13). We confirmed that recognition accuracy was not related to crop growth and the weed area could be almost perfectly segmented. It is fair to say that the method developed by combining the FL and GA was appropriate and effective.

Estimation of crop growth using an ANN

Crop growth information is important for making fertilizer application decisions as well as for investigating spatial variation in overall yield. A tractor operator qualitatively observes crop conditions during travel and operations in the field; an agricultural mobile robot also has to detect crop growth parameters such as crop height and width. A crop prediction method was developed for 


\section{N. NOGUCHI}

machine vision. An ANN was utilized to find the relationship between the image pattern and the crop growth parameters. The construction of the ANN is shown in Fig. 14. The ANN is a threelayer network consisting of an input layer, a hidden layer and an output layer. The gray levels of nine $20-\mathrm{cm}$ square tiles were the inputs to the ANN. To decide the center of the individual crop, $d_{5}$, which was the highest gray level in the neighbors, was adopted as a constraint condition. Training data were randomly chosen from the image data during the entire growth period. A back propagation algorithm (BP) was adopted as the ANN training method. A set of 300 training samples was used to construct the ANN. Figure 15 shows the ANN prediction accuracy for crop height and width using the training data. We investigated the relationship between measured and predicted values through the entire growth period. A total of 300 data sets were used for the investigation. The $r^{2}$ values for both crop height and crop width were 0.92 for the training data. It was concluded that the ANN could learn the crop size with a high level of accuracy. Figure 16 shows the results of investigation of prediction accuracy for test data that do not include the training data set. A total of 300 data sets were tested as well as the training data. The $r^{2}$ values for crop height and crop width were 0.832 and 0.849 for the test data, respectively. The high correlation implies that an $\mathrm{ANN}$ - machine vision system can be used as a crop prediction sensor.

\section{CONCLUSIONS}

In this paper, current AI technologies such as FL, ANN and GA were introduced and their impact on agricultural production systems was discussed. Various aspects of crop growth and development, such as bud formation, fruit set and fruit load, can be seen by the grower. But, it is

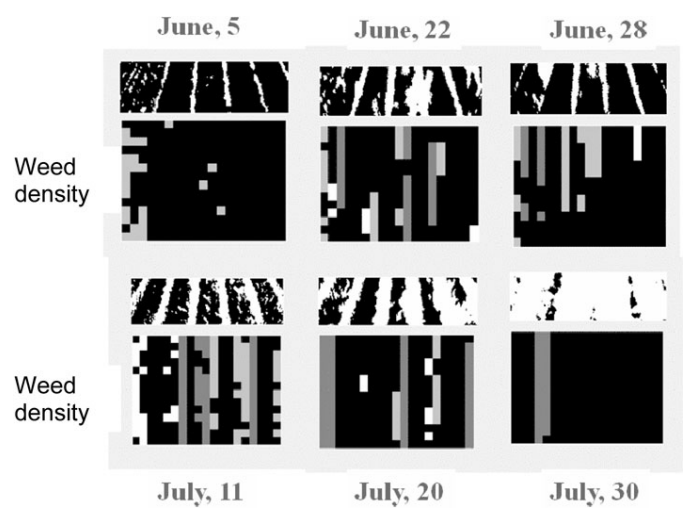

Fig. 13 Weed segmentation for six different growth stages through the entire growing period.

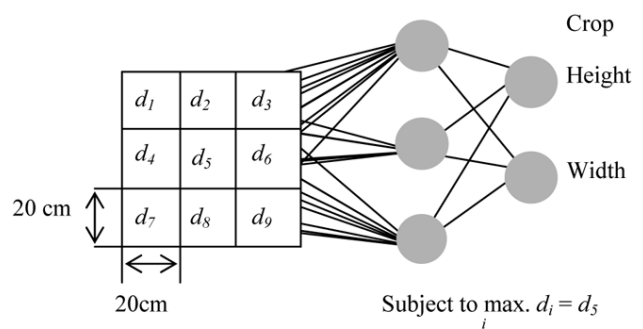

$d:$ density of vegetation

Fig. 14 Construction of the crop growth predictor using the ANN. 


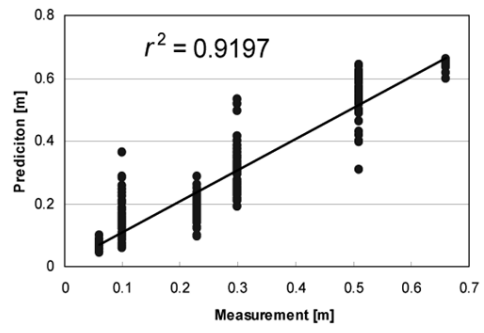

a) Height

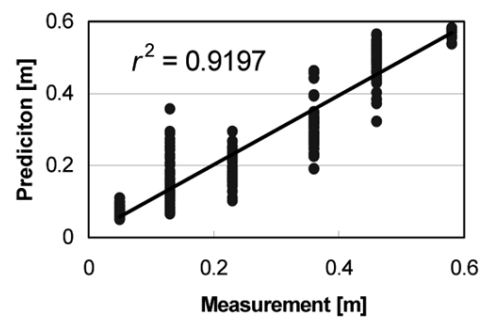

b) Width

Fig. 15 Accuracy of the ANN crop growth predictor for training data.

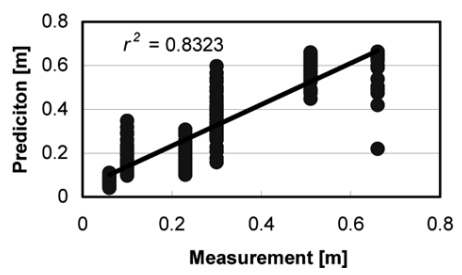

a) Height

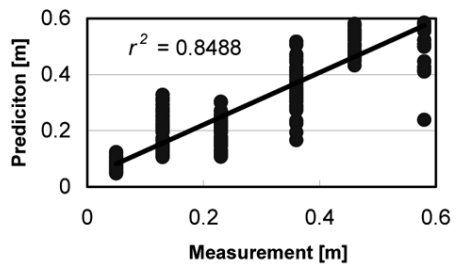

b) Width

Fig. 16 Accuracy of the ANN crop growth predictor for test data.

difficult to create a model of grower's decision making process. Therefore, an alternative approach involves trying to exploit the knowledge of the growers. This leads to rule-based approaches or to AI-based approaches using FL, ANN or GA. As an example of AI application for agricultural production systems, "smart vision" for a field robot was introduced in this paper. Machine vision that has FL optimized by a GA was developed to classify crops and weeds during field operations. Field tests confirmed that the classification method was accurate through the entire crop growth period. After segmenting out the weeds, the ANN is used to estimate crop height and width. 


\section{REFERENCES}

Carson, E., Feng, D. D., Pons, M., Soncini-Sessa, R., van Straten, G. 2008. Bio- and ecological systems: challenges, accomplishments and forecasts. Proceedings of the 17th IFAC World Congress: 3458-3469.

Hashimoto, Y., Morimoto, T., De Baerdemaeker, J. 2006. Speaking plant / speaking fruit approaches. CIGR Handbook of Agricultural Engineering VI : 244-259.

Holland, J. H. 1962. Outline for a logical theory of adaptive systems. Journal of the Association for Computing Machinery, 3: 297-314.

Holland, J. H. 1975. Adaptation in natural and artificial systems: University of Michigan Press, Ann Arbor, MI, pp 206.

Ichikawa, Y., Sano, T. 1992. Neural network application for direct feedback controller. IEEE Trans. Neural Networks, 3: 224-231.

Morimoto, Y., De Baerdemaeker, J., Hashimoto, Y. 1997. An intelligent approach for optimal control of fruit-storage process using neural networks and genetic algorithms. Comput. Electron. Agric. 18: 205-224.

Noguchi, N., Terao, H. 1997. Path planning of an agricultural mobile robot by neural network and genetic algorithm. Comput. Electron. Agric. 18: 187-204.

Noguchi, N., Reid, J. F., Zhang, Q., Tian, L., Hansen, A. C. 1999. Vision intelligence for mobile agro-robotic system. Journal of Robotics and Mechatronics, 11: 193-199.

Noguchi, N., Reid, J. F., Ishii, K., Terao, H. 2001. Multi-spectrum image sensor for detecting crop status by robot tractor. Proceedings of the Fourth IFAC Symposium on Intelligent Autonomous Vehicles: 111-115.

van Straten, G., van Willigenburg, L. G. 2008. On evaluating optimality losses of greenhouse climate controllers. Proceedings of the 17th IFAC World Congress: 2944-2949.

Zadeh, L. A. 1965. Fuzzy sets. Information Control, 8: 338-353. 